\title{
Treatment Outcome and Associated Factors among TB Patients in Ethiopia: Hospital-Based Retrospective Study
}

\author{
Girum Taye*, Atkure Defar, Tefera Taddele, Abebe Bekele, Theodros Getachew, Habtamu Teklie, \\ Geremew Gonfa, Misrak Getnet, Mengistu Tadesse, Minilik Demissie \\ Ethiopian Public Health Institute, P.o.Box 1242, Addis Ababa Ethiopia \\ *Corresponding author: girumt2000@yahoo.com
}

\begin{abstract}
Background: Tuberculosis remains a major global health problem and ranks alongside the human immunodeficiency virus (HIV) as a leading cause of mortality worldwide. For effective tuberculosis control, it is a pre-requisite to detect the cases as early as possible, and to ensure that the tuberculosis patients complete their treatment and get cured. However, in many resource-constrained settings treatment outcome for tuberculosis has not been satisfactory. Objective: The aim of the study was to assess the treatment outcome of tuberculosis and investigate the association of demographic and clinical factors with treatment success of patients enrolled in selected hospitals, Ethiopia. Methods: A fifteen-year retrospective register based historical data were collected through medical record review from 31 selected hospitals in Ethiopia. Data were analyzed using SPSS version 20 and to investigate the association of demographic and clinical factors with treatment success of patients, multiple logistic regression methods were used. A $p$ value of less than $5 \%$ was considered as statistically significant in the final model. Result: Out of the 90,191 registered tuberculosis patients (50,167 males and 40,024 females) including all age group, $55.8 \%$ had successful treatment outcome and $44.2 \%$ had unsuccessful outcome. In the multivariate logistic model, the odds of unsuccessful treatment outcome was relatively higher among patients in the age group of $\geq 65$ ( $\mathrm{AOR}=1.283$, 95\% CI: 1.186-1.387), unknown presumptive MDR TB (AOR=1.17, 95\% CI:1.07-1.279) and unknown smear result (AOR=1.706, 95\% CI:1.611-1.807); and was lower in age group 15-24 (AOR $=0.818,95 \%$ CI: 0.781-0.857), among female patients (AOR $=0.89,95 \%$ CI: 0..866-0.914) and extra pulmonary TB patients (AOR=0.889, 95\% CI:0.848-0.932 as compared to their respective comparison groups. Conclusion: In this study, high proportion of unsuccessful treatment outcome was documented, therefore emphasis has to be given for patients with high risk of unsuccessful TB treatment outcome and targeted interventions should be carried out. All patients' background characteristics were significantly associated with the treatment success status. And a continuous followup of patients with frequent supportive supervision during the course of treatment is recommended.
\end{abstract}

Keywords: Multivariate, logistic model, MDR TB, Adjusted Odds Ratio, TB treatment outcome

Cite This Article: Girum Taye, Atkure Defar, Tefera Taddele, Abebe Bekele, Theodros Getachew, Habtamu Teklie, Geremew Gonfa, Misrak Getnet, Mengistu Tadesse and Minilik Demissie, "Treatment Outcome and Associated Factors among TB Patients in Ethiopia: Hospital-Based Retrospective Study." American Journal of Epidemiology and Infectious Disease, vol. 6, no. 1 (2018): 14-19. doi: 10.12691/ajeid-6-1-3.

\section{Background}

According to WHO report, Ethiopia is among the 30 high burden countries for TB, TB/HIV and MDR-TB with estimated TB prevalence and incidence rates of 200 and 207 per 100,000 population in 2014 [1].Considering TB as one of the major public health challenges for the country, the FMOH has given due attention and included TB prevention and control among major priority programs [2]

Since 1997, much has been done to improve accessibility of TB diagnostic and treatment services to rural communities by equipping and staffing newly constructed primary health care units with microscopes, GeneXpert machines and trained health manpower $[3,4]$. Community based TB care intervention has been implemented as part of health extension program package since 2004, to decentralize TB case finding and treatment supervision to the level of health posts [5].

As it is has presented in the WHO on the Road to end TB document that, The End TB Strategy is not a "one size fits all" approach and its success depends on adaptation for diverse country settings. Thus country specific intervention is vital [6].

As a result, the country has achieved tuberculosis related MDG targets by halting and reversing the incidence rate from 369 in 1990 to 224 per 100,000 populations in 2014. It has also reduced TB prevalence and mortality rate by more than half compared to the 1990 baseline values.

Despite these achievements one-third of incident TB cases remained undetected and only a third of estimated drug-resistant TB cases are getting proper treatment every year [1]. Hence, Tuberculosis remains among the top ten causes of mortality in the country [4]. 
Cognizant of the facts, Ethiopia is required to exert much more effort to detect the missed one-third new TB cases and effectively treat at least $90 \%$ of notified TB cases. Treatment success rate (TSR) of TB patients was still low and a declining trend of TSR was observed [9].

Therefore, the study was conducted to assess the treatment outcome of tuberculosis and investigate the association of demographic and clinical factors with treatment success of patients enrolled in selected hospitals, Ethiopia.

\subsection{Specific Objectives}

1. To assess the trend of TB and treatment outcomes from the selected hospitals in the past fifteen years

2. To assess the outcomes of TB patients registered for anti-tuberculosis treatment in selected hospitals

3. To identify factors associated with treatment success for Tuberculosis patients in selected hospitals

\section{Methods}

The study was conducted in 31 selected hospitals from all regions and city administrative of Ethiopia. The data recorded from September-July, 2001/2 to 2015/16 were collected for the present study. The source population of the study was all patients registered for treatment of TB in the selected Hospitals. The study population was all tuberculosis patients who had treatment outcome at selected Hospitals between September-August, 2001/22015/16.

All data were retrieved from records of patient registration who took anti-TB treatment during the study period.

For the data collection from selected hospitals, EPHI identified and recruited health professionals working in the Outpatient Department TB clinics of the respective selected hospital as data collectors and the hospital head as supervisor/team leader besides frequent supervision made by EPHI Staff.

After data collection was completed, training has been given to the data entry team on the survey questionnaires, the nature of the data to be computerized, and the data entry template. Double data entry, cleaning and analyses were done using SPSS 20 statistical software. In order to control for possible errors during data entry, validation techniques such as supervision and running intermediate frequencies were employed.

To identify the factors for unsuccessful TB treatment outcome, study participants were categorized as having successful treatment if their record showed that they were cured or they had completed the treatment. Otherwise, they were categorized as treatment not successful (i.e. the record showed that the patient was either treatment failure, died, lost to follow-up, transferred out or not evaluated).

\section{Ethical Consideration}

The content of the proposal for the study on hospitals were reviewed and approved by scientific and ethical review committee of the Ethiopian Public Health Institute (EPHI) for its conformity with basic guidelines for ethical clearance of the Institute.

\section{Results}

\subsection{Patients' Demographic Characteristics}

As shown in Table 1, the high prevalence of all forms of TB cases observed among TB patients in the age group 25-34 (28.2\%) followed by 15-24 (25.3\%) and 35-49 (21.2\%). The least prevalence seen in the age group $>=65$ (3.7\%) followed by 50-64 (8.9\%) and <=14 (12.7\%).

Table 1. Socio-demographic characteristics of all Tuberculosis patients, in selected 31 hospitals Ethiopia 2001/2-2015/16

\begin{tabular}{|c|c|c|}
\hline Characteristics & Frequency & Percent \\
\hline \multicolumn{3}{|l|}{ Age } \\
\hline$\leq 14$ & 11442 & 12.7 \\
\hline $15-24$ & 22804 & 25.3 \\
\hline $25-34$ & 25451 & 28.2 \\
\hline $35-49$ & 19139 & 21.2 \\
\hline $50-64$ & 8001 & 8.9 \\
\hline$>=65$ & 3354 & 3.7 \\
\hline \multicolumn{3}{|l|}{ Sex } \\
\hline$\underline{\text { Male }}$ & 50167 & 55.6 \\
\hline$\underline{\text { Female }}$ & 40024 & 44.4 \\
\hline \multicolumn{3}{|l|}{ Type of TB } \\
\hline Pulmonary & 61400 & 68.1 \\
\hline Extra pulmonary & 28405 & 31.5 \\
\hline$\underline{\text { Unknown }}$ & 386 & 0.4 \\
\hline \multicolumn{3}{|l|}{ Presumptive MDR TB } \\
\hline$\underline{\text { Yes }}$ & 2190 & 2.4 \\
\hline$\underline{\text { No }}$ & 31749 & 35.2 \\
\hline Unknown & 56252 & 62.4 \\
\hline \multicolumn{3}{|l|}{ Smear result } \\
\hline$\underline{\text { Positive }}$ & 22272 & 24.7 \\
\hline Negative & 44407 & 49.2 \\
\hline Unknown & 23512 & 26.1 \\
\hline \multicolumn{3}{|l|}{ Outcome } \\
\hline Cured & 11981 & 13.3 \\
\hline Treatment Completed & 38306 & 42.5 \\
\hline Treatment Failure & 365 & 0.4 \\
\hline$\underline{\text { Died }}$ & 4924 & 5.5 \\
\hline$\underline{\mathrm{LTFU}}$ & 3654 & 4.1 \\
\hline$\underline{\text { Transferred out }}$ & 23659 & 26.2 \\
\hline$\underline{\text { Not evaluated }}$ & 7302 & 8.1 \\
\hline \multicolumn{3}{|l|}{ Year } \\
\hline$\underline{2001 / 2}$ & 7566 & 8.4 \\
\hline$\underline{2002 / 3}$ & 7086 & 7.9 \\
\hline$\underline{2003 / 4}$ & 6360 & 7.1 \\
\hline$\underline{2004 / 5}$ & 6406 & 7.1 \\
\hline$\underline{2005 / 6}$ & 6408 & 7.1 \\
\hline$\underline{2006 / 7}$ & 6256 & 6.9 \\
\hline$\underline{2007 / 8}$ & 4699 & 5.2 \\
\hline$\underline{2008 / 9}$ & 6154 & 6.8 \\
\hline$\underline{2009 / 10}$ & 6397 & 7.1 \\
\hline$\underline{2010 / 11}$ & 8272 & 9.2 \\
\hline$\underline{2011 / 12}$ & 6966 & 7.7 \\
\hline$\underline{2012 / 13}$ & 4658 & 5.2 \\
\hline$\underline{2013 / 14}$ & 4892 & 5.4 \\
\hline$\underline{2014 / 15}$ & 4552 & 5.0 \\
\hline$\underline{2015 / 16}$ & 3519 & 3.9 \\
\hline Total & \multicolumn{2}{|c|}{90191} \\
\hline
\end{tabular}

Moreover, greater proportion of TB cases revealed among male (55.6\%) TB patients compared to females (44.4\%).

Percentage distribution of TB by type shows, about 68 percent were pulmonary followed by extra pulmonary (31.5\%). And only 24.7 percent of all forms of TB cases 
were smear positive result.

The Figure 1 shows a trend of the proportion of all forms of TB cases. Highest proportion of TB was observed in
2010/11 (9.2\%) while the lowest was observed in 2015/16 (3.9\%). Generally, a decreasing trend observed from 2010/11 onwards.

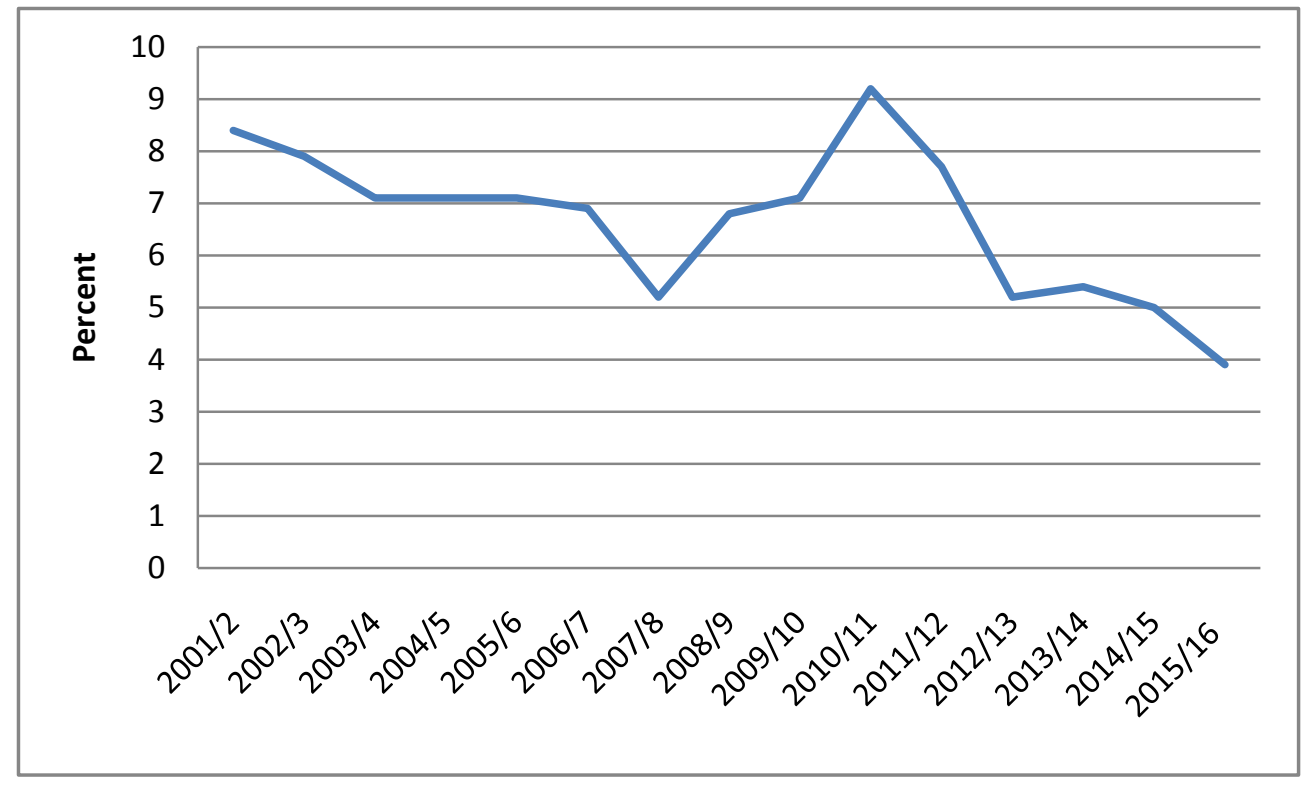

Figure 1. Trend of all forms of TB cases, in selected 31 hospitals in Ethiopia, 2001/2-2015/16

Table 2. Treatment outcomes of registered Tuberculosis patients, Ethiopia 2001/2-2015/16

\begin{tabular}{|c|c|c|c|c|c|c|c|}
\hline \multirow[b]{2}{*}{ Characteristics } & \multicolumn{7}{|c|}{ Treatment outcome } \\
\hline & $\begin{array}{l}\text { Cured } \\
\mathrm{n}(\%)\end{array}$ & $\begin{array}{l}\text { Treatment } \\
\text { Completed } \\
\mathrm{n}(\%)\end{array}$ & $\begin{array}{l}\text { Treatment } \\
\text { Failure } \\
\mathrm{n}(\%)\end{array}$ & $\begin{array}{l}\text { Died } \\
\mathrm{n}(\%)\end{array}$ & $\begin{array}{l}\text { LTFU } \\
\mathrm{n}(\%)\end{array}$ & $\begin{array}{l}\text { Transferred out } \\
\text { n(\%) }\end{array}$ & $\begin{array}{l}\text { Not evaluated } \\
\mathrm{n}(\%)\end{array}$ \\
\hline 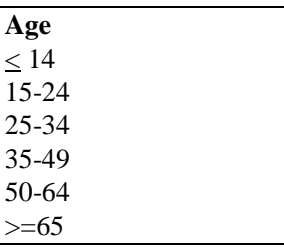 & $\begin{array}{l}494(4.3) \\
4118(18.1) \\
3939(15.5) \\
2398(12.5) \\
788(9.8) \\
244(7.3) \\
\end{array}$ & $\begin{array}{l}5746(50.2) \\
9836(43.1) \\
10254(40.3) \\
7770(40.6) \\
3322(41.5) \\
1378(41.1) \\
\end{array}$ & $\begin{array}{l}30(0.3) \\
95(0.4) \\
106(0.4) \\
82(0.4) \\
35(0.4) \\
17(0.5) \\
\end{array}$ & $\begin{array}{l}426(3.7) \\
745(3.3) \\
1524(6) \\
1407(7.4) \\
531(6.6) \\
291(8.7) \\
\end{array}$ & $\begin{array}{l}431(3.8) \\
781(3.4) \\
1061(4.2) \\
842(4.4) \\
357(4.5) \\
182(5.4) \\
\end{array}$ & $\begin{array}{l}3309(28.9) \\
5590(24.5) \\
6344(24.9) \\
5100(26.6) \\
2353(29.4) \\
963(28.7) \\
\end{array}$ & $\begin{array}{l}1006(8.8) \\
1639(7.2) \\
2223(8.7) \\
1540(8) \\
615(7.7) \\
279(8.3) \\
\end{array}$ \\
\hline $\begin{array}{l}\text { Sex } \\
\text { Male } \\
\text { Female } \\
\end{array}$ & $\begin{array}{l}6771(13.5) \\
5210(13) \\
\end{array}$ & $\begin{array}{l}20531(40.9) \\
17775(44.4)\end{array}$ & $\begin{array}{l}213(0.4) \\
152(0.4)\end{array}$ & $\begin{array}{l}2789(5.6) \\
2135(5.3) \\
\end{array}$ & $\begin{array}{l}2092(4.2) \\
1562(3.9) \\
\end{array}$ & $\begin{array}{l}13481(26.9) \\
10178(25.4)\end{array}$ & $\begin{array}{l}4290(8.6) \\
3012(7.5) \\
\end{array}$ \\
\hline $\begin{array}{l}\text { Type of TB } \\
\text { Pulmonary } \\
\text { Extra pulmonary } \\
\text { Unknown } \\
\end{array}$ & $\begin{array}{l}11592(18.9) \\
378(1.3) \\
11(2.8)\end{array}$ & $\begin{array}{l}23403(38.1) \\
14728(51.9) \\
175(45.3) \\
\end{array}$ & $\begin{array}{l}313(0.5) \\
48(0.2) \\
4(1)\end{array}$ & $\begin{array}{l}3458(5.6) \\
1446(5.1) \\
20(5.2) \\
\end{array}$ & $\begin{array}{l}2476(4) \\
1161(4.1) \\
17(4.4) \\
\end{array}$ & $\begin{array}{l}14991(24.4) \\
8546(30.1) \\
122(31.6) \\
\end{array}$ & $\begin{array}{l}5167(8.4) \\
2098(7.4) \\
37(9.6) \\
\end{array}$ \\
\hline $\begin{array}{l}\text { Presumptive MDR TB } \\
\text { Yes } \\
\text { No } \\
\text { Unknown } \\
\end{array}$ & $\begin{array}{l}548(25) \\
4745(14.9) \\
6688(11.9) \\
\end{array}$ & $\begin{array}{l}725(33.1) \\
13504(42.5) \\
24077(42.8) \\
\end{array}$ & $\begin{array}{l}40(1.8) \\
66(0.2) \\
259(0.5) \\
\end{array}$ & $\begin{array}{l}149(6.8) \\
1731(5.5) \\
3044(5.4) \\
\end{array}$ & $\begin{array}{l}46(2.1) \\
968(3) \\
2640(4.7) \\
\end{array}$ & $\begin{array}{l}601(27.4) \\
8476(26.7) \\
14582(25.9) \\
\end{array}$ & $\begin{array}{l}81(3.7) \\
2259(7.1) \\
4962(8.8) \\
\end{array}$ \\
\hline $\begin{array}{l}\text { Smear result } \\
\text { Positive } \\
\text { Negative } \\
\text { Unknown } \\
\end{array}$ & $\begin{array}{l}11056(49.6) \\
689(1.6) \\
236(1) \\
\end{array}$ & $\begin{array}{l}2834(12.7) \\
23570(53.1) \\
11902(50.6) \\
\end{array}$ & $\begin{array}{l}199(0.9) \\
125(0.3) \\
41(0.2) \\
\end{array}$ & $\begin{array}{l}1063(4.8) \\
2671(6) \\
1190(5.1) \\
\end{array}$ & $\begin{array}{l}744(3.3) \\
1944(4.4) \\
966(4.1) \\
\end{array}$ & $\begin{array}{l}4597(20.6) \\
11996(27) \\
7066(30.1) \\
\end{array}$ & $\begin{array}{l}1779(8) \\
3412(7.7) \\
2111(9) \\
\end{array}$ \\
\hline $\begin{array}{l}\text { Year } \\
2001 / 2 \\
2002 / 3 \\
2003 / 4 \\
2004 / 5 \\
2005 / 6 \\
2006 / 7 \\
2007 / 8 \\
2008 / 9 \\
2009 / 10 \\
2010 / 11 \\
2011 / 12 \\
2012 / 13 \\
2013 / 14 \\
2014 / 15 \\
2015 / 16 \\
\end{array}$ & $\begin{array}{l}1210(16) \\
1169(16.5) \\
977(15.4) \\
793(12.4) \\
691(10.8) \\
712(11.4) \\
614(13.1) \\
788(12.8) \\
714(11.2) \\
844(10.2) \\
832(11.9) \\
697(15) \\
669(13.7) \\
746(16.4) \\
525(14.9)\end{array}$ & $\begin{array}{l}3167(41.9) \\
3025(42.7) \\
2433(38.3) \\
2917(45.5) \\
2801(43.7) \\
3092(49.4) \\
2268(48.3) \\
2682(43.6) \\
2723(42.6) \\
3307(40) \\
2593(37.2) \\
2085(44.8) \\
2021(41.3) \\
2101(46.2) \\
1091(31)\end{array}$ & $\begin{array}{l}30(0.4) \\
27(0.4) \\
32(0.5) \\
24(0.4) \\
28(0.4) \\
12(0.2) \\
24(0.5) \\
20(0.3) \\
30(0.5) \\
39(0.5) \\
30(0.4) \\
15(0.3) \\
37(0.8) \\
12(0.3) \\
5(0.1)\end{array}$ & $\begin{array}{l}530(7) \\
476(6.7) \\
489(7.7) \\
439(6.9) \\
422(6.6) \\
334(5.3) \\
298(6.3) \\
359(5.8) \\
337(5.3) \\
362(4.4) \\
258(3.7) \\
186(4) \\
147(3) \\
151(3.3) \\
136(3.9)\end{array}$ & $\begin{array}{l}339(4.5) \\
348(4.9) \\
294(4.6) \\
368(5.7) \\
453(7.1) \\
381(6.1) \\
155(3.3) \\
318(5.2) \\
148(2.3) \\
161(1.9) \\
121(1.7) \\
187(4) \\
176(3.6) \\
156(3.4) \\
49(1.4)\end{array}$ & $\begin{array}{l}1287(17) \\
1206(17) \\
1244(19.6) \\
1565(24.4) \\
1474(23) \\
1276(20.4) \\
1011(21.5) \\
1501(24.4) \\
1986(31) \\
2873(34.7) \\
2634(37.8) \\
1328(28.5) \\
1697(34.7) \\
1258(27.6) \\
1319(37.5)\end{array}$ & $\begin{array}{l}1003(13.3) \\
835(11.8) \\
891(14) \\
300(4.7) \\
539(8.4) \\
449(7.2) \\
329(7) \\
486(7.9) \\
459(7.2) \\
686(8.3) \\
498(7.1) \\
160(3.4) \\
145(3) \\
128(2.8) \\
394(11.2)\end{array}$ \\
\hline Total & 11981(13.3) & $38306(42.5)$ & $365(0.4)$ & $4924(5.5)$ & $3654(4.1)$ & $23659(26.2)$ & $7302(8.1)$ \\
\hline
\end{tabular}




\subsection{Treatment Outcome}

Of 90,191 TB patients who were registered at selected hospitals during the study period, 1,198 (13.3\%) were cured, 38,306(42.5\%) completed their treatment, 365 (0.4\%) were with treatment failure, 4,924 (5.5\%) died, 3654 (4.1\%) lost to follow up, 23,659 (26.2\%) were transferred out to other health facility and 7,302(8.1\%) were not evaluated. The cure rate of TB patients varied from $4.3 \%$ in age group $<=14$ to $18.1 \%$ in age group $15-24$ (Table 2).

\subsection{Type of TB}

Except TB patients in age group $<=14$ (55.1\%), in all other age groups about $70 \%$ of TB patients were infected with pulmonary TB. The proportion of pulmonary TB is relatively high among males (69.2\%) compared to females (66.7\%) TB patients.

Generally, in all TB patients background characteristics pulmonary TB was more prevalent compared to extra pulmonary TB. Although, Pulmonary TB is higher than extra pulmonary TB, higher proportion of were observed in the lower age group $(\leq 14)$ compared with the other age groups.

\subsection{Factors Associated with Treatment Outcomes}

As shown in Table 4 below, status of treatment successful outcome was significantly associated with patients' age group, sex, type of TB, presumptive MDR $\mathrm{TB}$, smear result and year of registration (P-value $<0.05$ ) with successful and unsuccessful treatment outcome proportion of $55.8 \%$ and $44.2 \%$, respectively.

The unsuccessful treatment outcome was 1.283, 1.167, 1.116 times more likely among TB patients in age group $\geq$ $65,50-64$ and 35-49, respectively compared to patients in age group $<=14$.

With unknown presumptive MDR TB the unsuccessful treatment outcome was 1.17 times more likely compared to TB patients with presumptive MDR TB. The odd of unsuccessful treatment outcome was 1.347 times more likely among TB patients with negative smear result compared to TB patients with positive smear result.

Among Female patients unsuccessful treatment outcome was 11 percent less likely compared to male patients. The unsuccessful treatment outcome was about 11 percent less likely among extra pulmonary TB patients compared to pulmonary.

Table 3. Characterristics of registered TB patients in 31 selected hospitals, Ethiopia 2001/2-2015/16

\begin{tabular}{|c|c|c|c|}
\hline \multirow{2}{*}{ Characteristics } & \multicolumn{3}{|c|}{ Type of TB } \\
\hline & Pulmonary & Extra Pulmonary & Unknown \\
\hline \multicolumn{4}{|l|}{ Age } \\
\hline$<14$ & $6310(55.1)$ & $5069(44.3)$ & $63(0.6)$ \\
\hline$\overline{1} 5-24$ & 15581(68.3) & 7141(31.3) & $82(0.4)$ \\
\hline $25-34$ & 17961(70.6) & 7404(29.1) & $86(0.3)$ \\
\hline $35-49$ & $13569(70.9)$ & $5468(28.6)$ & $102(0.5)$ \\
\hline $50-64$ & $5631(70.4)$ & 2332(29.1) & $38(0.5)$ \\
\hline$>=65$ & $2348(70)$ & $991(29.5)$ & $15(0.4)$ \\
\hline \multicolumn{4}{|l|}{ Sex } \\
\hline Male & $34696(69.2)$ & 15262(30.4) & 209(0.4) \\
\hline Female & $26704(66.7)$ & $13143(32.8)$ & $177(0.4)$ \\
\hline \multicolumn{4}{|l|}{ Presumptive MDR TB } \\
\hline Yes & 1823(83.2) & $357(16.3)$ & $10(0.5)$ \\
\hline No & 20258(63.8) & 11422(36) & $69(0.2)$ \\
\hline Unknown & 39319(69.9) & 16626(29.6) & $307(0.5)$ \\
\hline \multicolumn{4}{|l|}{ Smear result } \\
\hline Positive & 21882(98.2) & $367(1.6)$ & $23(0.1)$ \\
\hline Negative & $37921(85.4)$ & $6316(14.2)$ & $170(0.4)$ \\
\hline Unknown & $1597(6.8)$ & 21722(92.4) & $193(0.8)$ \\
\hline \multicolumn{4}{|l|}{ Outcome } \\
\hline Cured & 11592(96.8) & $378(3.2)$ & $11(0.1)$ \\
\hline Treatment completed & 23403(61.1) & 14728(38.4) & $175(0.5)$ \\
\hline Treatment failure & $313(85.8)$ & $48(13.2)$ & $4(1.1)$ \\
\hline Died & $3458(70.2)$ & $1446(29.4)$ & $20(0.4)$ \\
\hline LTFU & $2476(67.8)$ & 1161(31.8) & $17(0.5)$ \\
\hline Transferred out & 14991(63.4) & 8546(36.1) & $122(0.5)$ \\
\hline Not evaluated & $5167(70.8)$ & 2098(28.7) & $37(0.5)$ \\
\hline \multicolumn{4}{|l|}{ Year } \\
\hline $2001 / 2$ & $5438(71.9)$ & 2052(27.1) & 76(1) \\
\hline 2002/3 & $4778(67.4)$ & 2292(32.3) & $16(0.2)$ \\
\hline $2003 / 4$ & $4272(67.2)$ & 2051(32.2) & $37(0.6)$ \\
\hline $2004 / 5$ & $4283(66.9)$ & 2120(33.1) & $3(0)$ \\
\hline 2005/6 & $4307(67.2)$ & 2076(32.4) & $25(0.4)$ \\
\hline 2006/7 & $4517(72.2)$ & $1719(27.5)$ & 20.(0.3) \\
\hline 2007/8 & $3315(70.5)$ & 1369(29.1) & $15(0.3)$ \\
\hline 2008/9 & $4235(68.8)$ & 1881(30.6) & $38(0.6)$ \\
\hline 2009/10 & $4336(67.8)$ & 2015(31.5) & $46(0.7)$ \\
\hline 2010/11 & $5074(61.3)$ & $3164(38.2)$ & $34(0.4)$ \\
\hline 2011/12 & $4493(64.5)$ & 2461(35.3) & $12(0.2)$ \\
\hline 2012/13 & 3330(71.5) & 1318(28.3) & $10(0.2)$ \\
\hline 2013/14 & $3413(69.8)$ & $1466(30)$ & $13(0.3)$ \\
\hline 2014/15 & $3166(69.6)$ & 1361(29.9) & $25(0.5)$ \\
\hline 2015/16 & 2443(69.4) & $1060(30.1)$ & $16(0.5)$ \\
\hline
\end{tabular}


Table 4. Treatment outcomes by TB patients background characteristics, Ethiopia 2001/2-2015/16

\begin{tabular}{|c|c|c|c|c|}
\hline \multirow[b]{2}{*}{ Characteristics } & \multicolumn{2}{|l|}{ Treatment outcome } & \multicolumn{2}{|l|}{ Adjusted Odds Ratio } \\
\hline & Unsuccessful n (\%) & $\begin{array}{l}\text { Successful } \\
n(\%)\end{array}$ & AOR(95\% C.I) & P-value \\
\hline $\begin{array}{l}\text { Age } \\
\leq 14 \\
15-24 \\
25-34 \\
35-49 \\
50-64 \\
>=65 \\
\end{array}$ & $\begin{array}{l}5202(45.5) \\
8850(38.8) \\
11258(44.2) \\
8971(46.9) \\
3891(48.6) \\
1732(51.6) \\
\end{array}$ & $\begin{array}{l}6240(54.5) \\
13954(61.2) \\
14193(55.8) \\
10168(53.1) \\
4110(51.4) \\
1622(48.4) \\
\end{array}$ & $\begin{array}{l}1 \\
0.818(0.781,0.857) \\
1.027(0.982,1.075) \\
1.116(1.064,1.170) \\
1.167(1.101,1.237) \\
1.283(1.186,1.387) \\
\end{array}$ & $\begin{array}{l}<0.001 \\
0.242 \\
<0.001 \\
<0.001 \\
<0.001\end{array}$ \\
\hline $\begin{array}{l}\text { Sex } \\
\text { Male } \\
\text { Female } \\
\end{array}$ & $\begin{array}{l}22865(45.6) \\
17039(42.6)\end{array}$ & $\begin{array}{l}27302(54.4) \\
22985(57.4)\end{array}$ & $\begin{array}{l}1 \\
0.89(0.866,0.914)\end{array}$ & $\begin{array}{l}1 \\
<0.001 \\
\end{array}$ \\
\hline $\begin{array}{l}\text { Type of TB } \\
\text { Pulmonary } \\
\text { Extra pulmonary } \\
\text { Unknown } \\
\end{array}$ & $\begin{array}{l}26405(43) \\
13299(46.8) \\
200(51.8)\end{array}$ & $\begin{array}{l}34995(57) \\
15106(53.2) \\
186(48.2)\end{array}$ & $\begin{array}{l}1 \\
0.889(0.848,0.932) \\
1.129(0.921,1.383)\end{array}$ & $\begin{array}{l}1 \\
<0.001 \\
0.243\end{array}$ \\
\hline $\begin{array}{l}\text { Presumptive MDR TB } \\
\text { Yes } \\
\text { No } \\
\text { Unknown }\end{array}$ & $\begin{array}{l}917(41.9) \\
13500(42.5) \\
25487(45.3)\end{array}$ & $\begin{array}{l}1273(58.1) \\
18249(57.5) \\
30765(54.7)\end{array}$ & $\begin{array}{l}1 \\
1.004(0.918,1.098) \\
1.170(1.07,1.279)\end{array}$ & $\begin{array}{l}1 \\
0.939 \\
0.001\end{array}$ \\
\hline $\begin{array}{l}\text { Smear result } \\
\text { Positive } \\
\text { Negative } \\
\text { Unknown } \\
\end{array}$ & $\begin{array}{l}8382(37.6) \\
20148(45.4) \\
11374(48.4)\end{array}$ & $\begin{array}{l}13890(62.4) \\
24259(54.6) \\
12138(51.6)\end{array}$ & $\begin{array}{l}1 \\
1.347(1.301,1.394) \\
1.706(1.611,1.807)\end{array}$ & $\begin{array}{l}1 \\
<0.001 \\
<0.001\end{array}$ \\
\hline $\begin{array}{l}\text { Year } \\
2001 / 2 \\
2002 / 3 \\
2003 / 4 \\
2004 / 5 \\
2005 / 6 \\
2006 / 7 \\
2007 / 8 \\
2008 / 9 \\
2009 / 10 \\
2010 / 11 \\
2011 / 12 \\
2012 / 13 \\
2013 / 14 \\
2014 / 15 \\
2015 / 16 \\
\end{array}$ & $\begin{array}{l}3189(42.1) \\
2892(40.8) \\
2950(46.4) \\
2696(42.1) \\
2916(45.5) \\
2452(39.2) \\
1817(38.7) \\
2684(43.6) \\
2960(46.3) \\
4121(49.8) \\
3541(50.8) \\
1876(40.3) \\
2202(45) \\
1705(37.5) \\
1903(54.1) \\
\end{array}$ & $\begin{array}{l}4377(57.9) \\
4194(59.2) \\
3410(53.6) \\
3710(57.9) \\
3492(54.5) \\
3804(60.8) \\
2882(61.3) \\
3470(56.4) \\
3437(53.7) \\
4151(50.2) \\
3425(49.2) \\
2782(59.7) \\
2690(55) \\
2847(62.5) \\
1616(45.9) \\
\end{array}$ & $\begin{array}{l}1 \\
0.923(0.864,0.986) \\
1.171(1.094,1.253) \\
0.996(0.93,1.066) \\
1.143(1.069,1.223) \\
0.867(0.810,0.929) \\
0.867(0.805,0.935) \\
1.05(0.980,1.124) \\
1.189(1.111,1.273) \\
1.364(1.28,1.453) \\
1.425(1.333,1.523) \\
0.935(0.867,1.008) \\
1.146(1.065,1.234) \\
0.852(0.789,0.920) \\
1.724(1.588,1.872) \\
\end{array}$ & $\begin{array}{l}1 \\
0.018 \\
<0.001 \\
0.901 \\
<0.001 \\
<0.001 \\
<0.001 \\
0.165 \\
<0.001 \\
<0.001 \\
<0.001 \\
0.079 \\
<0.001 \\
<0.001 \\
<0.001\end{array}$ \\
\hline Total & 39904(44.2) & 50287(55.8) & & \\
\hline
\end{tabular}

\section{Discussion}

As a major indicator for the evaluation of the performance of a national TB program, assessment of anti-tuberculosis treatment outcome and identifying factors associated with unsuccessful treatment outcome is crucial. In this hospital based historical cohort study, information was extracted from 90191 registered TB patients; $44.4 \%$ of the patients were females. Similarly, previous study at University of Gondar Teaching Hospital documented the same proportion of female patients registered for TB treatment [7]. In this study the successful outcome proportion is found to be $55.8 \%$ while it is $60.1 \%$ in the previous study at Gondar Teaching Hospital, and this difference could be due to the inclusion of transfer-out TB patient as unsuccessful treatment outcome in this study.

The $0.4 \%$ treatment failure and 5.5\% death rate documented in our findings are lower than Gondar teaching hospital, where $0.8 \%$ failed treatment and $17.7 \%$ had died.

Low rate of unsuccessful TB treatment was observed in the patient record review done in Metema hospital (34.7\%) [9]. Similarly, a study done in Gambella hospital revealed $29.3 \%$, this could be due to the fact that the study doesn't include transfer out cases [10]. Very low proportion of unfavorable outcome was observed in the other study done in Addis Ababa Health facilities which revealed closer proposition of failure to success to the WHO standard (17.8\%) [11] and 12.9\% had unsuccessful treatment outcome [12]. Furthermore, in western oromia the overall mean treatment rate of fail to success TB patients was $14.8 \%$ and $26 \%$ had bad outcome [13] and in another study failure of TB treatment fount to be $29 \%$ and $26 \%$, respectively [14,15]. Generally the lower and higher difference in failure to success on TB treatment from this current study was observed due to possibly methodological and geographical factors. A bit comparable result has been observed on the unfavorable outcome (39.9\%) on the study done in Gonder referral hospital [16].

In multivariable logistic regression the unsuccessful treatment outcomes of our finding were significantly higher in age groups $\geq 65,50-64$ and 35-49 ; and among smear negative TB patients compared to patients in age group $<=14$ and TB patients with positive smear result, respectively. The unsuccessful TB treatment outcome was significantly lower among female and extra pulmonary TB patients compared to male and pulmonary TB patients, respectively.

Since this study is based on secondary data analysis, it has limitations as well as strengths which need to be noted while interpreting the findings. The main strengths of the study is covering 31 hospitals from all regions of the country by assessing the available fifteen years records. 
The main limitation of this study like in other low income countries is the poor quality of reports [8].

In this study age, sex, type of TB were identified as factors associated with the result of treatment outcome, similarly being male, and co-infection were significantly associated with unsuccessful treatment of TB [9], this is because HIV co-infection is predominated among males whereas, co-infection with HIV and being male were associated with unsuccessful treatment outcome. On the other hand Tuberculosis type, age greater than 45, Baseline CD4 count less than 20cell/ul were factors associated with unsuccessful treatment outcome [14]. Being residing in outside the town, having less than the mean baseline weight $(<43.7 \mathrm{~kg})$ at initiation of $\mathrm{TB}$ treatment, being in the bedridden condition and experiencing anti-TB treatment side effect were the factors that resulted the patient in treatment failure [16]. HIV co-infected patients were associated with unsuccessful treatment outcomes [15]. Smear positive pulmonary TB and TB/HIV co-infections were significantly associated with unsuccessful treatment outcome [17].

\section{Conclusion}

The overall TB treatment success rate for all registered patients was $55.8 \%$ with high proportion of unsuccessful treatment outcome of $44.2 \%$. In addition to this, the following risk factors were identified as potential predictors for unsuccessful treatment outcome: patients' age, sex, type of TB, status of presumptive MDR TB and smear result. According to the findings of this study, emphasis should be given for patients with high risk of unsuccessful treatment outcome and targeted interventions need to be carried out.

\section{Acknowledgements}

The authors would like to acknowledge individuals who contributed to this study directly or indirectly in which without their support it could not have been possible to be productive. The Global Fund is acknowledged for financially supporting of this study.

\section{Conflict of Interests}

No conflict of interests between authors

\section{References}

[1] WHO: Global Tuberculosis Report 2015, $20^{\text {th }}$ edition

[2] FMOH of Ethiopia: Revised strategic plan Tuberculosis, TB/HIV, MDR TB, and Leprosy prevention and control 2006 - 2013 EC (2013/14 - 2020); October 2013 Unpublished, October 2013.

[3] FMOH: Health and health related indicators, 2008 EFY (2015/2016), October 2016

[4] FMOH: Annual TB Bulletin 2015. An extract of TB, TB/HIV and Leprosy control Program Analysis, volume 6 No. 6, March 2015.

[5] WHO country office for Ethiopia: Towards A Healthier Ethiopia; Achieving the health MDG; 2015, June 2015.

[6] H. From and B. Countries, "ON THE ROAD TO ENDING TB HIGHLIGHTS FROM THE 30 HIGHEST TB THE END TB STRATEGY : AT A GLANCE.”

[7] Minaleshewa Biruk et al: Treatment outcomes of Tuberculosis and associated factors in an Ethiopian University hospital; Hindawi Publishing Corporation Volume 2016.

[8] WHO: Tuberculosis control program resolution WHA44.8. Fortyfourth World Health Assembly. Handbook of resolutions and decisions of the World Health Assembly and the Executive Board, vol. III, $3^{\text {rd }}$ ed. (1985-1992) Geneva: WHO: WHO document WHA44/1991/REC/1; 1993.

[9] M. Jemal et al., "Mycobacterial Diseases Treatment Outcomes of Tuberculosis Patients in Metema Hospital , Northwest Ethiopia : A Four Years Retrospective Study,” vol. 5, no. 4, 2015.

[10] G. Asebe, H. Dissasa, T. Teklu, G. Gebreegizeabhe, K. Tafese, and G. Ameni, "Treatment outcome of Tuberculosis Patients at Gambella Hospital, Southwest Ethiopia : Three-year Retrospective Study,” vol. 3, no. 2, pp. 1-7, 2015.

[11] "Treatment outcome of tuberculosis patients under directly_Addis Aba."

[12] A. Melese, B. Zeleke, and B. Ewnete, "Treatment Outcome and Associated Factors among Tuberculosis Patients in Debre Tabor , Northwestern Ethiopia : A Retrospective Study,” vol. 2016, 2016.

[13] C. Open and A. Journal, "Treatment Outcome of Tuberculosis and Associated Factors at Gimbi Town Health Facilities Western Oromia ," vol. 2, no. 2, 2017.

[14] M. Belayneh, K. Giday, and H. Lemma, "Original article Treatment outcome of human immunodeficiency virus and tuberculosis co-infected patients in public hospitals of eastern and southern zone of Tigray region , Ethiopia,” Brazilian J. Infect. Dis., vol. 19, no. 1, pp. 47-51, 2014.

[15] B. Wondale, G. Medihn, T. Teklu, W. Mersha, M. Tamirat, and G. Am, "RESEARCH NOTE A retrospective study on tuberculosis treatment outcomes at Jinka General Hospital , southern Ethiopia,” pp. 1-7, 2017.

[16] M. Biruk, B. Yimam, H. Abrha, S. Biruk, and F. Z. Amdie, "Treatment Outcomes of Tuberculosis and Associated Factors in an Ethiopian University Hospital,” vol. 2016, 2016.

[17] D. Mekonnen, A. Derbie, H. Mekonnen, Y. Zenebe, and D. Mekonnen, "Profile and treatment outcomes of patients with tuberculosis in Northeastern Ethiopia: a cross sectional study.," vol. 16, no. 3, pp. 663-670, 2016. 